by Butterworth, Sturrock, Houba and Rees (Nature, 252, 503; 1974). These authors found that schistosomula were damaged in the presence of normal leukocytes and sera from immune patients. The nature of the cells and antibodies involved is not disclosed but it is suggested that cytotoxicity is associated with eosinophils.

So far neutrophils and eosinophils have been suggested as effector cells in the immune response but the most recent work in this field also implicates macrophages. Capron, Bazin, Dessaint and Capron (Nature, 253, 474; 1974) found that normal rat macrophages adhered to schistosomula in the presence of immune sera in vitro. Absorption of the immune sera with various anti-rat-immunoglobulin sera showed the immunoglobulin involved to be IgE. Further experiments showed that the IgE was specific to the parasite, that macrophage adherence played a part in the killing of schistosomula and that complement was not required for the killing. Killing requires the presence of specific IgE which binds normal macrophages to the surface of the worm and, although it may contribute directly to the death of the worm, the main importance of this binding is that it is instrumental in enhancing the lethal effects of immune IgG. These experiments draw attention to the possibility of IgE-mediated macrophage adherence in other situations and at last provide a central rather than a peripheral role for IgE in immunity to helminth infections. Perhaps we will soon know what $\operatorname{IgE}$ really does other than cause asthma and hay fever.

\section{Peptidoglycan and the immune response}

\section{from a Correspondent}

THE bacterial envelope is comprised of the cytoplasmic membrane and the outer cell wall. The classic division of bacteria on the basis of the Gram stain turns out to correspond exactly to variations in the chemistry and structural organisation of the outer part of the envelope. But in spite of the variations there is a unique component, the peptidoglycan, present in greater or lesser amount in the cell wall of all bacteria. The peptidoglycan matrix is built up from strands of chitin-like polysaccharides but with every alternate sugar residue being $\mathrm{N}$-acetylmuramic acid (the 3-O-D-lactic acid ether of $\mathrm{N}$-acetylglucosamine). The acid residues are substituted by amide linkages with short peptide side chains and many of these chains are joined to link adjacent polysaccharide strands together in a meshwork. In most-per- haps all-gram negative bacteria and in several gram positive bacilli the peptidoglycan structures differ chemically only in detail although it seems probable that their three dimensional arrangements vary considerably. Outside this range of organisms the chemical differences, particularly in peptide structure, multiply enormously.

All this has been known for several years. It is therefore surprising that so little is known about the effects of the ubiquitous peptidoglycan on mammalian cells. Most people are in contact with bacterial peptidoglycan from very early life. Certainly peptidoglycan is immunogenic and there must be a continuous stimulation of the immune response by bacteria indigenous to the respiratory and gastrointestinal tracts.

And peptidoglycan is of course a component of complete Freund's adjuvant-the suspension of tubercle bacilli which, injected as a water-in-air emulsion with antigen, greatly enhances the immune response to many antigens. Does peptidoglycan play any part in adjuvanticity? It now seems clear that it does.

Purified peptidoglycan enhances antibody production and induces delayedtype hypersensitivity at rather lower concentrations than those of the whole bacilli used in Freund's adjuvant. There is a fascinating argument going on between two French laboratories about the minimum structural features of peptidoglycan necessary for the adjuvant effect. Lederer and his colleagues Adam, Ciorboru Ellouz and Petit (Biochem. biophys. Res. Commun., 59, 1317-1325; 1974) have taken degradation fragments of peptidoglycan from Mycobacterium semeqmatis or Escherichia coli and found that a monomeric fragment $\mathrm{N}$-acetylglucosaminyl $(\beta 1,4) \mathrm{N}$-acetylmuramyl - L alanyl - D - isoglutaminyl - meso - diaminopimelyl-D-alanine can replace whole killed mycobacteria or undegraded peptidoglycan. Furthermore the terminal $\mathrm{N}$-acetylglucosaminyl and D-alanyl units can be removed without loss of adjuvanticity. A synthetic compound, N-acetylmuramyl-L-alanylD-isoglutamine turns out to be at least as active as the monomeric unit but a synthetic disaccharide-L-alanyl derivative is inactive. So are the hexosamine free di- to tetra peptides. Lederer concludes that the glycopeptide linkage and $\mathrm{D}$-isoglutaminyl unit are indispensable for an adjuvant effect.

Nauciel and Fleck (C. r. Acad. Sci. Paris, 276D, 3499-3500; 1973; Nature, 250, 517-518; 1974; Eur. J. Immun., 4, $352-356$; 1974) disagree on the first point but support the importance of $\mathrm{D}$ isoglutamine. They find that the peptide subunit alone possesses the same adjuvant activity as intact peptidoglycan at least in delayed-type hypersensitivity reactions to azobenzenearsonate- $\mathrm{N}$ acetyl-L-tyrosine.

In a search for other points in the immune response at which peptidoglycan might function, the inhibitory effect of this substance on macrophage migration (Heymer et al., J. Immun., 116, 1743-1754; 1973) is relevant. This effect seems to be distinct from migration inhibition of macrophages mediated immunologically by factors secreted by activated lymphocytes. A direct action of peptidoglycan on the macrophage surface is possible but little is known as yet about the structural features responsible for this interesting effect.

Finally, stimulation of graft rejection -for example of tumour cells-by Mycobacterium tuberculosis BCG has been studied by Zbar et al., (J. natn. Cancer Inst., 48, 831-835; 1972), using isolated mycobacterial cell walls. The effect is complex and, at least in the systems in which virally induced tumours were studied, stimulation of host interferon production by the mycobacterial adjuvant is possible. But this presumably is not involved in host rejection of chemically induced tumours. Could a lectin-like activity of antibodies directed against the polysaccharide part of peptidoglycan be involved in host reactions against tumour cells? Several lectins that agglutinate tumour cells (for example, wheat germ agglutinin: Allen and Neuberger, Biochem. J., 135, 307-314; 1973) interact with an N-acetylchitobiose unit that is found commonly in mammalian glycoproteins, and also bind bacterial peptidoglycan containing an analogous repeating disaccharide unit. In some interesting experiments Shier (Proc. natn. Acad. Sci. U.S.A., 68, 2078-2082; 1971) prepared antisera against a synthetic antigen containing $\mathrm{N}$-acetylchitobiose hapten groups. His finding that these antibodies show some antitumour effects therefore raises the interesting question whether antibodies of similar specificity raised in response to peptidoglycan mimic this reactivity.

\section{Single-particle states of nuclei}

\section{from $P$. E. Hodgson}

$\mathrm{MuCH}$ of our knowledge of atomic nuclei has been obtained by studies of reactions that remove one nucleon from the nucleus or add one nucleon to it. Analysis of the cross sections of these interactions gives the quantum numbers and the occupation probabilities of the single-particle states of the simple shell model. This is a very basic feature of nuclei, and it is related to many other nuclear properties such 